\title{
Successful Treatment of Brevibacterium Bacteremia Solely With Antimicrobial Therapy
}

\author{
Charles E. Benson Jr. ${ }^{1}$, Luis Tatem ${ }^{1}$ \\ 1. Infectious Diseases, State University of New York Downstate Medical Center, New York, USA
}

Corresponding author: Luis Tatem, luis.tatem@downstate.edu

\begin{abstract}
Brevibacterium is a large genus that is not often involved in pathogenesis, however, since 1991 there have been several case reports of Brevibacterium-associated illness, most often due to bacteremia in the setting of an immunocompromised patient with a central venous catheter (CVC). Here we detail the case of an elderly woman with many comorbidities and a peripherally inserted central catheter (PICC) line for over four years, who presented with septic shock and Brevibacterium bacteremia. In nearly all previous cases of Brevibacterium bacteremia it was thought to be due to a CVC which was removed as part of the treatment in conjunction with antibiotics. In this case, the patient was treated with empiric antibiotics and her blood cultures cleared within 48 hours without catheter removal or antibiotic-lock therapy. The clinical outcome was favorable at 50 days follow-up.
\end{abstract}

Categories: Infectious Disease

Keywords: brevibacterium, bacteremia, central venous catheter infection, immunocompromised, picc line

\section{Introduction}

The genus of Brevibacterium contains 50 different species, nine of which have been isolated from humans, including B. casei (most common), B. epidermidis, B. otitidis, B. paucivorans, B. sanguinis, B. linens, B. iodinum, B. mcbrellneri, and $B$. massiliense [1]. Though this genus has been found on human skin, it was thought to be apathogenic until the discovery of a patient with Brevibacterium bacteremia in 1991. Since then, various case reports of bacteremia most often associated with central venous catheters in patients with malignancy or with immunocompromised conditions have been published. Although uncommon, they are important and under-recognized etiologic agents of infections in immunocompromised patients [2].

\section{Case Presentation}

An 85-year-old female with a past medical history of cholangitis and common bile duct (CBD) stones, who has had a percutaneous cholecystostomy tube (PCT) since 2018 due to her high surgical risk, heart failure reduced ejection fraction (HFrEF) on home intravenous milrinone via right subclavian peripherally inserted central catheter (PICC) (Figure 1) which was placed four years prior and never exchanged, implantable cardioverter defibrillator (ICD), coronary artery disease, aortoiliac aneurysm and mural thrombi, presented with altered mental status and fever of $100.9 \mathrm{~F}$. Initial vitals showed a heart rate of $132 \mathrm{bpm}$, systolic BP of $100 \mathrm{~mm} \mathrm{Hg}$, and an $\mathrm{SpO} 2$ of $91 \%$ on room air which improved to $95 \%$ with $15 \mathrm{~L}$ of nonrebreather. On exam she was noted to be disoriented, mildly dyspneic and had decreased air entry bilaterally, her right-sided PICC line site was clean without erythema, induration, or discharge, same as with her ICD site. Heart sounds were positive without any murmurs, abdominal exam showed leakage around her PCT and decreased drainage into the biliary collecting bag. She initially received two liters of intravenous fluids, was started on a norepinephrine drip due to worsening hemodynamic instability and received $2 \mathrm{~g}$ of ceftriaxone plus 500 milligrams of metronidazole. A set of blood cultures were obtained, a urinalysis was negative for leukocyte esterase or nitrates, and her white blood cell count was $7.45 \mathrm{~K} / \mathrm{mL}$ with $25 \%$ bands on manual differential. A cholangiogram was done showing the catheter to have withdrawn into the common bile duct (Figure 2). The CBD was moderately dilated with several filling defects consistent with calculi and a biliary drainage catheter exchange was done. Computer tomography of the abdomen showed enhancement of gallbladder wall, but the family declined endoscopic retrograde cholangiopancreatography (ERCP) and declined PICC line exchange. She was given vancomycin, cefepime, and metronidazole for empirical coverage of her sepsis syndrome as she was clinically unstable and was continued on norepinephrine support. Bile cultures from a previous drainage system showed Aeromonas, Escherichia coli, Klebsiella pneumoniae, Enterococcus faecalis, and Enterococcus faecium deemed to be colonizers as they were obtained from the drainage system, the polymicrobial nature of the specimen and as she had clinically stabilized despite the lack E. faecium coverage. Initial blood cultures revealed Brevibacterium species in the aerobic bottle after 96 hours of incubation, identified with matrix-assisted laser desorption ionization-time of flight mass spectrometry (MALDI-TOF MS) and a follow-up blood culture in 24 hours again showed a similar isolate. Antibiotics were switched to ceftriaxone $2 \mathrm{~g}$ daily plus metronidazole to cover for her gallbladder infection and she was continued on intravenous vancomycin empirically for Brevibacterium bacteremia. After 48 hours her condition completely stabilized, and cultures cleared on the third day of admission. She received a total of 10 days of intravenous vancomycin and this was followed by oral doxycycline as she was 


\section{Cureus}

allergic to penicillins; quinolones were not ideal due to her advanced age and aortic aneurysm. The family did not want another IV access and her PICC line was exclusively used for her milrinone continuous infusion, therefore this non-standard approach was performed. Brevibacterium antimicrobial susceptibility became available after discharge: Benzyl penicillin MIC $0.50 \mathrm{ug} / \mathrm{ml}$ intermediate, ciprofloxacin MIC $0.47 \mathrm{ug} / \mathrm{ml}$ susceptible, erythromycin MIC $0.50 \mathrm{ug} / \mathrm{ml}$ susceptible, gentamicin MIC $0.064 \mathrm{ug} / \mathrm{ml}$ susceptible, tetracycline MIC $16 \mathrm{ug} / \mathrm{ml}$ resistant, trimethoprim/sulfamethoxazole $>32 \mathrm{ug} / \mathrm{ml}$ resistant, and vancomycin $0.50 \mathrm{ug} / \mathrm{ml}$ susceptible, according to the Clinical and Laboratory Standards Institute guidelines for corynebacteria [3]. We decided to stop her antibiotics seven days after discharge as the isolate was resistant to tetracyclines and follow her closely. She remains stable 60 days after discharge without relapse of her Brevibacterium bacteremia and without the need for a catheter exchange, without requiring further antibiotic therapy; after two months catheter was exchanged over the wire as per family request.

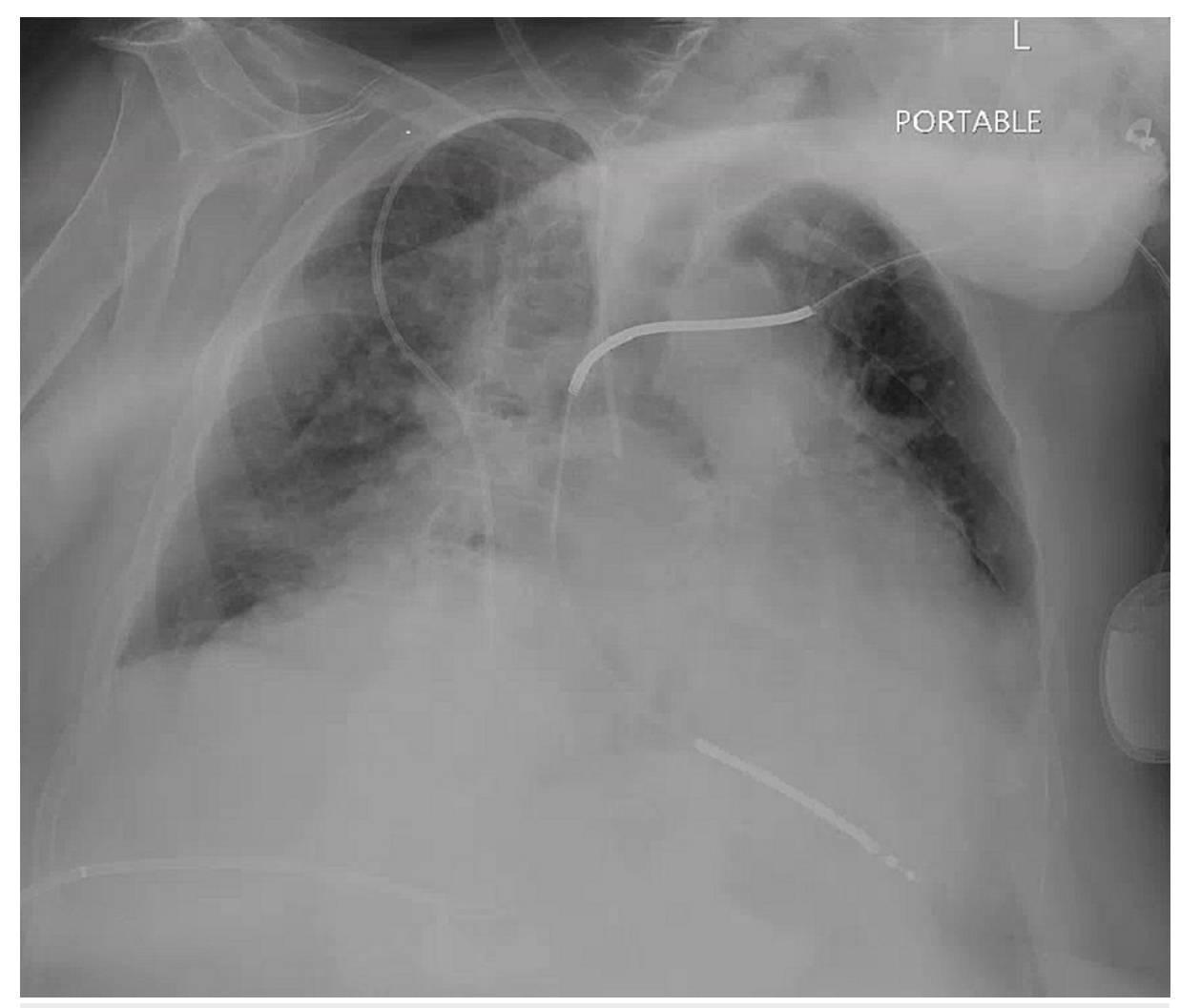

FIGURE 1: Portable chest X-ray antero-posterior (AP) 


\section{Cureus}

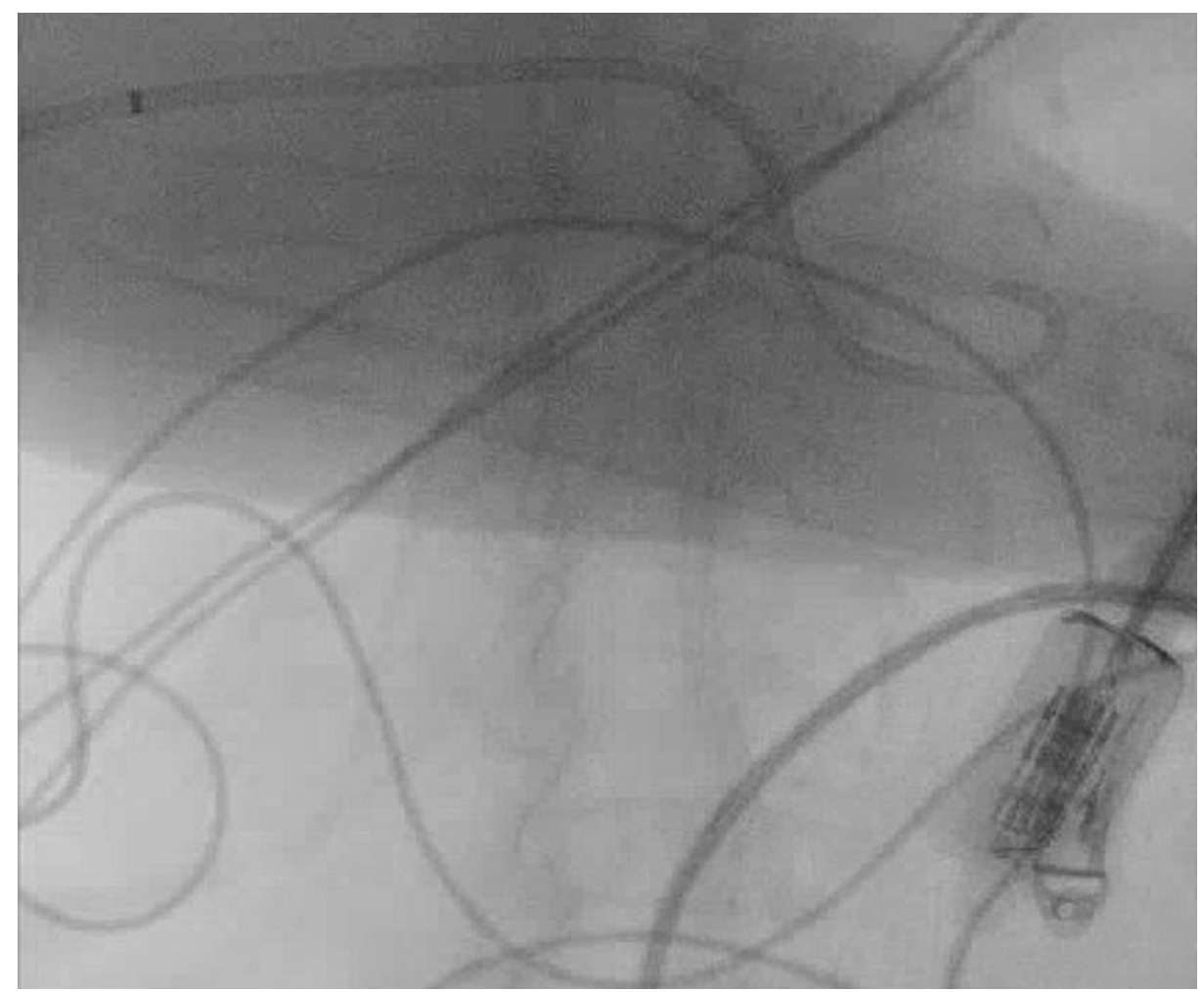

\section{FIGURE 2: Cholangiogram showing the percutaneous cholecystostomy tube (PCT) withdrawn}

\section{Discussion}

Brevibacterium are gram-positive rods, obligate aerobic organisms that are irregular and resemble corynebacteria. They are most often associated with milk products where they contribute to aroma and color but they are also found on human skin, genital hair, and otorrhea [2]. Brevibacterium is rarely a pathogenic genus, the first case not being discovered until 1991 when B. epidermidis was found to be the cause of a central line-associated bloodstream infection [4]. Since this discovery, it has infrequently been found as a pathogen with a total of 18 cases reported implicating Brevibacterium, the most common infection being bacteremia (10 of these cases). The most common species that is isolated is B. casei [5]. There have also been cases of peritonitis, pericarditis, endocarditis, brain abscess, and osteomyelitis [5-17]. As Asai et al. reported, the cases of Brevibacterium bacteremia most often occur as opportunistic pathogens in patients who are immunocompromised, usually due to malignancy or AIDS. Other important risk factors include indwelling foreign materials, prosthetic heart valves, and continuous ambulatory peritoneal catheters for dialysis [18]. There is also an overwhelming correlation between bacteremia by this genus and indwelling central venous catheters (CVC) with a CVC being present in 10 out of 11 cases of bacteremia [2]. Of the cases in which bacteremia is associated with indwelling catheters, all of them were treated with antibiotics for two to three weeks, predominantly with glycopeptides and quinolones - as well as with removal of the patient's catheter, with some studies recommending this as a preferred part of the treatment, especially in patients with complicated bacteremia $[17,19]$.

It has also been shown in at least one patient that antibiotic-lock therapy with vancomycin, combined with intravenous antibiotic therapy was enough to clear Brevibacterium in a patient with uncomplicated bacteremia due to a Hickman catheter [19]. Most of the patients for which the information is available improved with antibiotic therapy and catheter removal, however it is important to note that in one study, $30 \%$ of patients had a recurrence of bacteremia from 13 to 28 days [2]. Our patient, while not immunocompromised, had multiple comorbidities and a long-standing CVC. By administering intravenous vancomycin, she was able to clear her bacteremia in two days without the need for catheter removal or antibiotic-lock therapy. Even though her cultures were not obtained directly from the catheter, the fact that the PCT cultures did not isolate the same organism and the presence of a long standing PICC line (over four years) makes this source the most plausible etiology of the Brevibacterium bacteremia. The fact that she remains stable 60 days post-discharge makes a catheter retention strategy as an alternative in patients needing to keep their access on a long-term basis and who have poor tolerance for repeated surgical procedures or patient's refusal.

\section{Conclusions}


Brevibacterium bacteremia, although uncommon, has now been increasingly reported, especially in patients with malignancy or with immunocompromised conditions who have central venous access. In most of the previous cases in literature, removal of the patient's central venous access is a paramount step in their management. Here we report a case in which Brevibacterium bacteremia was successfully treated solely with intravenous vancomycin for 10 days, with the patient remaining stable at 60 days follow-up without relapse.

\section{Additional Information \\ Disclosures}

Human subjects: Consent was obtained or waived by all participants in this study. Conflicts of interest: In compliance with the ICMJE uniform disclosure form, all authors declare the following: Payment/services info: All authors have declared that no financial support was received from any organization for the submitted work. Financial relationships: All authors have declared that they have no financial relationships at present or within the previous three years with any organizations that might have an interest in the submitted work. Other relationships: All authors have declared that there are no other relationships or activities that could appear to have influenced the submitted work.

\section{References}

1. Vecten M, Gouriet F, Cano A, Raoult D: Brevibacterium massiliense bacteremia. IDCases. 2017, 7:25-26. 10.1016/j.idcr.2016.11.010

2. Asai N, Suematsu H, Yamada A, et al.: Brevibacterium paucivorans bacteremia: case report and review of the literature. BMC Infect Dis. 2019, 19:344. 10.1186/s12879-019-3962-y

3. Clinical and Laboratory Standards Institute: Methods for Antimicrobial Dilution and Disk Susceptibility Testing of Infrequently Isolated or Fastidious Bacteria. Clinical and Laboratory Standards Institute, Wayne, PA; 2016.

4. McCaughey C, Damani NN: Central venous line infection caused by Brevibacterium epidermidis . J Infect. 1991, 23:211-212. 10.1016/0163-4453(91)92451-a

5. Eidensohn Y, Wei A, Sirkin M, Dever LL: Brevibacteria tibial osteomyelitis . IDCases. 2021, 23:e01046 10.1016/j.idcr.2021.e01046

6. Lina B, Carlotti A, Lesaint V, Devaux Y, Freney J, Fleurette J: Persistent bacteremia due to Brevibacterium species in an immunocompromised patient. Clin Infect Dis. 1994, 18:487-488. 10.1093/clinids/18.3.487

7. Reinert RR, Schnitzler N, Haase G, Lütticken R, Fabry U, Schaal KP, Funke G: Recurrent bacteremia due to Brevibacterium casei in an immunocompromised patient. Eur J Clin Microbiol Infect Dis. 1995, 14:10821085. 10.1007/BF01590943

8. Kaukoranta-Tolvanen SS, Sivonen A, Kostiala AA, Hormila P, Vaara M: Bacteremia caused by Brevibacterium species in an immunocompromised patient. Eur J Clin Microbiol Infect Dis. 1995, 14:801804. 10.1007/BF01690997

9. Brazzola P, Zbinden R, Rudin C, Schaad UB, Heininger U: Brevibacterium casei sepsis in an 18-year-old female with AIDS. J Clin Microbiol. 2000, 38:3513-3514. 10.1128/JCM.38.9.3513-3514.2000

10. Wauters G, Van Bosterhaut B, Avesani V, Cuvelier R, Charlier J, Janssens M, Delmée M: Peritonitis due to Brevibacterium otitidis in a patient undergoing continuous ambulatory peritoneal dialysis. J Clin Microbiol. 2000, 38:4292-4293. 10.1128/JCM.38.11.4292-4293.2000

11. Oğünç D, Gültekin M, Colak D, et al.: Bacteremia caused by Brevibacterium species in a patient with chronic lymphocytic leukemia. Haematologia (Budap). 2002, 32:151-153. 10.1163/156855902320387998

12. Janda WM, Tipirneni P, Novak RM: Brevibacterium casei bacteremia and line sepsis in a patient with AIDS .J Infect. 2003, 46:61-64. 10.1053/jinf.2002.1076

13. Dass KN, Smith MA, Gill VJ, Goldstein SA, Lucey DR: Brevibacterium endocarditis: a first report. Clin Infect Dis. 2002, 35:20-21. 10.1086/340984

14. Cannon JP, Spandoni SL, Pesh-Iman S, Johnson S: Pericardial infection caused by Brevibacterium casei. Clin Microbiol Infect. 2005, 11:164-165. 10.1111/j.1469-0691.2004.01050.x

15. Ulrich S, Zbinden R, Pagano M, Fischler M, Speich R: Central venous catheter infection with Brevibacterium sp. in an immunocompetent woman: case report and review of the literature. Infection. 2006, 34:103-106. 10.1007/s15010-006-5027-6

16. Kumar VA, Augustine D, Panikar D, Nandakumar A, Dinesh KR, Karim S, Philip R: Brevibacterium casei as a cause of brain abscess in an immunocompetent patient. J Clin Microbiol. 2011, 49:4374-4376. 10.1128/JCM.01086-11

17. Magi B, Migliorini L, Sansoni A, Cusi MG: Brevibacterium casei bacteraemia in a port-a-cath carrier patient: a case report and literature review. Infez Med. 2018, 26:263-265.

18. Olender A, Rutyna P, Niemcewicz M, Bogut A, Ciesielka M, Teresiński G: Draft whole-genome sequence of Brevibacterium casei strain isolated from a bloodstream infection. Braz J Microbiol. 2020, 51:685-689. 10.1007/s42770-020-00236-X

19. Beukinga I, Rodriguez-Villalobos H, Deplano A, Jacobs F, Struelens MJ: Management of long-term catheterrelated Brevibacterium bacteraemia. Clin Microbiol Infect. 2004, 10:465-467. 10.1111/j.14690691.2004.00857.x 